\title{
乙烯基碳上的亲核取代反应
}

\author{
沈舒苏 \\ (苏州科技学院分离净化材料与技术研发中心 苏州 215009)
}

\begin{abstract}
摘要 综述了近二十年来关于 $\mathrm{sp}^{2}$ 杂化碳, 主要是乙烯基碳上发生的亲核取代反应的研究进展. 讨论了乙烯基碳上的亲 核取代反应的理论支持，阐明了其在有机合成中，特别是在杂环化合物的合成中的重要应用. 同时还总结了其他不饱 和原子如 $\mathrm{sp}^{2}$ 杂化氮的亲核取代反应的研究以及应用.
\end{abstract}

关键词 乙烯基碳; $\mathrm{sp}^{2}$ 杂化碳; 亲核取代反应; $\mathrm{sp}^{2}$ 杂化氮

\section{Nucleophilic Substitution Reactions at Vinylic Carbons}

\author{
Shen, Shusu \\ $\left({ }^{a}\right.$ Center for Separation and Purification Materials \& Technologies, Suzhou University of \\ Science and Technology, Suzhou 215009)
}

\begin{abstract}
The recently research progress in the nucleophilic substitution reactions at the $\mathrm{sp}^{2}$-carbons, mainly at the vinylic carbons is reviewed. The theoretical studies on the nucleophilic substitution reactions at the vinylic carbons, and their applications in organic synthesis, especially in the synthesis of heterocycle compounds, are illustrated. And, researches and applications of the nucleophilic substitution reactions at other unsaturated atoms like $\mathrm{sp}^{2}$ nitrogens are also discussed.
\end{abstract}

Keywords vinylic carbon; $\mathrm{sp}^{2}$ carbon; nucleophilic substitution reaction; $\mathrm{sp}^{2}$ nitrogen

亲核取代反应 ${ }^{[1]}$ 在有机化学中是一类非常基础的反 应，被广泛地应用于有机合成当中. 在过去的几十年中， 关于亲核取代反应的机理研究已经相当地成熟. 尤其是 当亲核取代反应发生在一个饱和的原子上, 比如 $\mathrm{sp}^{3}-\mathrm{C}$ $\left(\mathrm{sp}^{3} \text { 杂化的碳原子), } \mathrm{S}_{\mathrm{N}} 1 \text { (单分子亲核取代反应) }\right)^{[2]}$ 和 $\mathrm{S}_{\mathrm{N}} 2$ (双分子亲核取代反应) ${ }^{[3]}$ 这两种反应机理是最常见 的两类.

对于不饱和碳原子, 例如 $\mathrm{sp}^{2}-\mathrm{C}$ ( $\mathrm{sp}^{2}$ 杂化碳), 虽然 关于 $S_{N} A r$ 反应(芳环上的亲核取代反应) 已经研究的比 较成熟 ${ }^{[4]}$, 但是, $\mathrm{S}_{\mathrm{N}} \mathrm{V}$ 反应(乙烯基碳上的亲核取代反应) 却长期以来一直被忽视, 至少在基础有机化学课本中被 认为是不可能发生的反应. 其中一个理由认为 $\mathrm{sp}^{2}-\mathrm{C}$ 的 电负性要高于 $\mathrm{sp}^{3}-\mathrm{C}$, 进而使得 $\mathrm{sp}^{2}-\mathrm{C}-\mathrm{X}$ 的 $\mathrm{C}-\mathrm{X}$ (碳卤)键的键能要大于 $\mathrm{sp}^{3}-\mathrm{C}-\mathrm{X}$ 的键能. 例如, $\mathrm{sp}^{2}-\mathrm{C}-\mathrm{Cl}$ 键的键长 $(1.73 \AA)$ 要小于 $\mathrm{sp}^{3}-\mathrm{C}-\mathrm{Cl}$ 键的键长 $(1.78 \AA)$, 这意味着 $\mathrm{sp}^{2}-\mathrm{C}-\mathrm{Cl}$ 键中氯原子的离去能力相对要小(图 $1)^{[5]}$.

尽管在乙烯基碳上发生亲核取代反应曾经被认为 是很难发生的, 迄今, 有机化学界仍有不少相关的实验

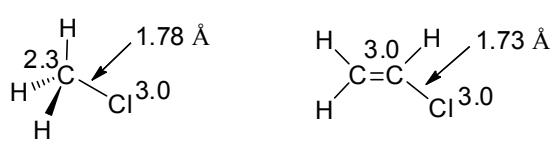

图 $1 \mathrm{sp}^{3}-\mathrm{C}-\mathrm{X}$ 与 $\mathrm{sp}^{2}-\mathrm{C}-\mathrm{X}$ 的键长

Figure 1 Bond-lengths of $\mathrm{sp}^{3}-\mathrm{C}-\mathrm{X}$ and $\mathrm{sp}^{2}-\mathrm{C}-\mathrm{X}$

事实以及机理推测的相关研究发表. 但是, 关于 $S_{N} V$ 反 应的研究主要以国外学者研究居多, 国内课题组对于此 类反应的研究鲜有报道. 本文主要结合作者在博士课程 期间在分子内 $\mathrm{S}_{\mathrm{N}} \mathrm{V}$ 反应的相关研究, 从机理讨论, 理论 计算以及实验论证等方面对乙烯基碳上的亲核取代反 应进行综述.

\section{1 机理方面的研究}

$\mathrm{sp}^{3}-\mathrm{C}$ 上的亲核取代反应基本上被分为 $\mathrm{S}_{\mathrm{N}} 1$ 和 $\mathrm{S}_{\mathrm{N}} 2$ 机理，而对于 $\mathrm{sp}^{2}-\mathrm{C}$ 上发生的亲核取代反应的机理则分 类比较多, 这是由于 $\mathrm{sp}^{2}-\mathrm{C}$ 有两个空轨道: $\sigma$ *轨道 $(\sigma$ 反键 轨道)和 $\pi *$ 轨道( $\pi$ 反键轨道), 因而目前化学界主要将此 类反应的机理分为以下这四种类型 ${ }^{[6]}$.

第一个类型从严格意义上来讲并非直接的亲核取

* E-mail: shusushen@mail.usts.edu.cn

Received June 27, 2014; revised July 17, 2014; published online August 11, 2014. 
代反应，而是一个亲核加成一消除反应 $\left(\mathrm{Ad}_{\mathrm{N}}-\mathrm{E}\right)$ (Scheme 1a). 这一机理一般适用于底物中的离去基团 $\mathrm{Y}^{-}$是一个 较强的吸电子基团的时候, 即底物中的碳碳双键被吸电 子基团活化. 在这个历程中, 亲核试剂 $\mathrm{Nu}^{-}$首先从垂直 的或者是平面外的方向进攻 $\mathrm{sp}^{2}-\mathrm{C}$ 的 $\pi$ *轨道, 进而生成 一个被吸电子基团稳定住的碳负离子的中间体. 这个中 间体紧接着发生离去基团的 $\beta$-消除而生成取代产物. 这 样一个两步历程被称为亲核加成一消除机理. 一般来说, 为了使得碳负离子中间体能够稳定地形成, $\mathrm{Ad}_{\mathrm{N}} \mathrm{E}$ 机理 常见于含有较强的吸电子基团的活泼烯烃上发生的取 代反应.
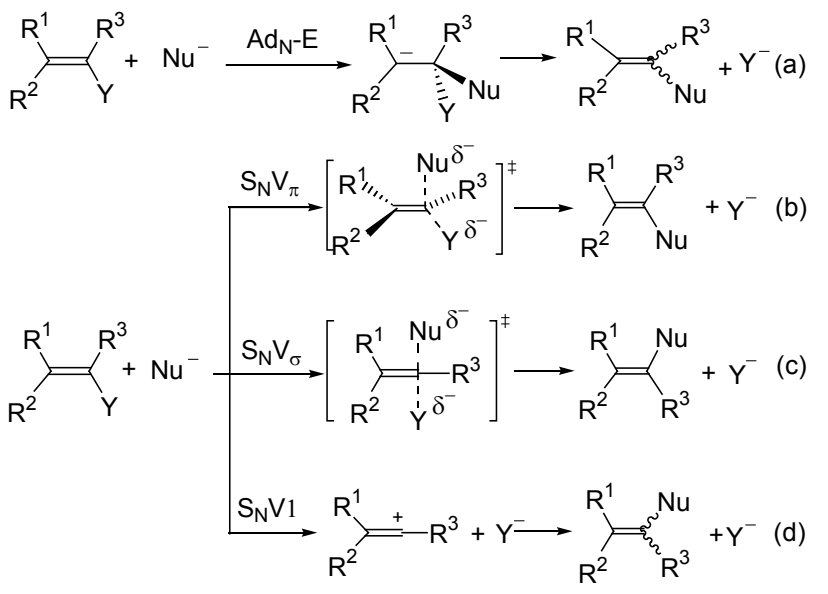

Scheme 1

那么, 对于不活泼的烯烃上发生的亲核取代反应则 经历其他的反应机理. 在 $\mathrm{Ad}_{\mathrm{N}} \mathrm{-E}$ 机理中, 随着取代基团 $\mathrm{R}^{1}$ 和 $\mathrm{R}^{2}$ 的吸电子能力的逐渐减弱或者是离去基团 $\mathrm{Y}^{-}$的 离去能力增强, 碳负离子中间体的寿命会变得很短, 从 而使得反应以协同的方式进行, 也就是说, 亲核试剂的 进攻与离去基团的离去同时发生. 这种情况被称为 $\mathrm{S}_{\mathrm{N}} \mathrm{V}_{\pi}$ (发生于乙烯基碳的 $\pi$ *轨道的亲核取代)机理, 在这 个反应过程之后, 产物一般保持立体构型不变(Scheme $1 b)$.

相应地, 另一种机理是 $\mathrm{S}_{\mathrm{N}} \mathrm{V}_{\sigma}$ (发生于乙烯基碳的 $\sigma^{*}$ 轨道的亲核取代)机理: 亲核试剂进攻 $\mathrm{C}-\mathrm{Y}$ 键的 $\sigma$ *轨 道, 意即在烯烃的双键平面内从离去基团的背面进攻. 这样一个平面内进攻, 可以令产物的构型产生翻转
(Scheme 1c).

当离去基团 $\mathrm{Y}^{-}$的离去能力很强时, 比如 $\mathrm{TfO}^{-}$(三氟 甲基磺酸根), 此时发生的反应历程与 $\mathrm{sp}^{3}-\mathrm{C}$ 上发生的 $\mathrm{S}_{\mathrm{N}} 1$ 机理很相似: 首先是发生 $\mathrm{C}-\mathrm{Y}$ 键的断裂, 生成乙 烯基碳正离子，进而被亲核试剂进攻，因此，此种历程 被称作 $\mathrm{S}_{\mathrm{N}} \mathrm{V} 1$ (乙烯基碳的单分子亲核取代)机理(Scheme 1d).

\section{2 乙烯基碳上的协同亲核取代反应的理论计算}

通过以上四种机理的讨论可以看出, 对于 $\mathrm{sp}^{2}-\mathrm{C}$ 上 发生的协同进行的亲核取代反应，主要适用于 $\mathrm{S}_{\mathrm{N}} \mathrm{V}_{\pi}$ 和 $\mathrm{S}_{\mathrm{N}} \mathrm{V}_{\sigma}$ 这两种机理. $\mathrm{MO}$ (分子轨道)理论一般认为, $\sigma^{*}$ 轨道 的能量通常是高于 $\pi^{*}$ 轨道的. 那么, 从理论上来说, 亲 核试剂 $\mathrm{Nu}^{-}$进攻 $\mathrm{sp}^{2}-\mathrm{C}$ 的 $\pi^{*}$ 轨道应该比进攻 $\sigma^{*}$ 轨道应该 相对容易. 因而，一般认为发生在 $\mathrm{sp}^{2}-\mathrm{C}$ 上的协同亲核 取代反应经历 $\mathrm{S}_{\mathrm{N}} \mathrm{V}_{\pi}$ 机理比 $\mathrm{S}_{\mathrm{N}} \mathrm{V}_{\sigma}$ 更容易. 然而, 事实上 并不是所有反应都遵循这样的规律, 关于 $\mathrm{S}_{\mathrm{N}} \mathrm{V}$ 反应的一 些理论计算的报道与这个假设结论是相反的.

对于发生在不活泼的乙烯基卤化物上的取代反应 有以下一些理论计算的报道 ${ }^{[7]} .1994$ 年, Glukhovtsev 等 ${ }^{[8]}$ 经过理论计算得出氯离子对1-氯乙烯的平面内亲核 取代 $\left(\mathrm{S}_{\mathrm{N}} \mathrm{V}_{\sigma}\right)$ 反应的活化能为 $136.9 \mathrm{~kJ} \cdot \mathrm{mol}^{-1}$, 而平面外 亲核取代 $\left(\mathrm{S}_{\mathrm{N}} \mathrm{V}_{\pi}\right)$ 反应所需的活化能则为 $179.3 \mathrm{~kJ} \cdot \mathrm{mol}^{-1}$, 可以看出, 后者比前者的活化能高出了约 $42 \mathrm{~kJ} \cdot \mathrm{mol}^{-1}$

(Scheme 2).

2000 年, Lee 课题组 ${ }^{[9]}$ 通过计算发现, 在气相下发生 亲核进攻氯乙烯时，氢氧根离子 $\left(9.0 \mathrm{~kJ} \cdot \mathrm{mol}^{-11}\right)$ 或者硫 氢根离子 $\left(102.3 \mathrm{~kJ} \cdot \mathrm{mol}^{-1}\right)$ 经历的是 $\mathrm{S}_{\mathrm{N}} \mathrm{V}_{\pi}$ 机理; 而氯离 子 $\left(125.0 \mathrm{~kJ} \cdot \mathrm{mol}^{-1}\right)$ 或溴离子 $\left(134.9 \mathrm{~kJ} \cdot \mathrm{mol}^{-1}\right)$ 作为亲核 试剂时, 则被认为经历了 $\mathrm{S}_{\mathrm{N}} \mathrm{V}_{\sigma}$ 的历程(各自的过度态所 需活化能均相对较低)(Scheme 3).

从以上两篇理论计算的结论中可以看出: 如果仅在 温和的实验条件下, 无论是哪种乙烯基碳的亲核取代反 应都不容易进行, 因为它们所需的反应的活化能都比较 高. 但是, 通过寻找一种适当的实验条件, $\mathrm{S}_{\mathrm{N}} \mathrm{V}$ 反应是 应该是可以实现的.

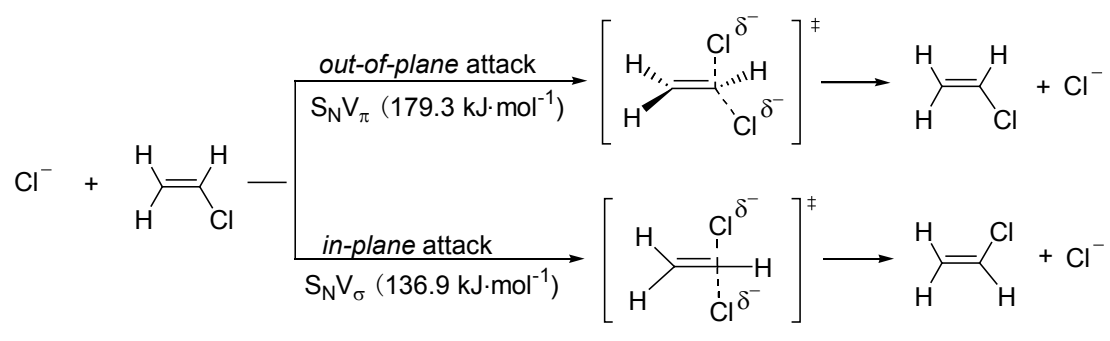

Scheme 2 


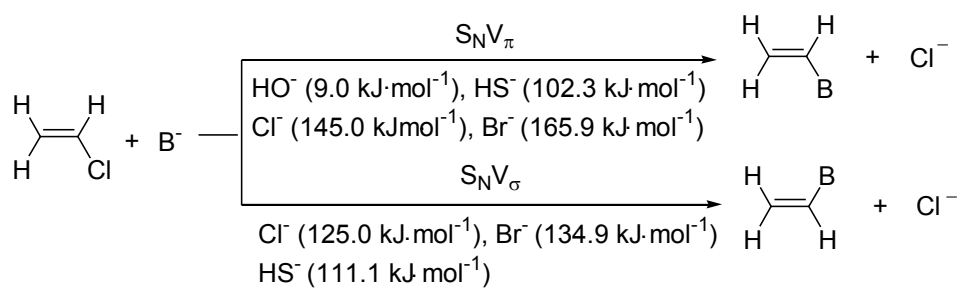

Scheme 3

\section{3 乙烯基碳上的亲核取代反应}

\section{1 活泼的乙烯基碳上的亲核取代反应}

在 1968 年, Marchese 等 ${ }^{[10]}$ 报道了一对异构体, $Z$ 式 和 $E$ 式两种构型的 $\beta$-卤代苯乙烯与硫负离子发生的分 子间亲核取代反应. 尽管反应的立体选择性不是很好, 但是生成的产物大部分还是以构型保持为主(Scheme 4). 当使用硒负离子作为亲核试剂的时候, 取代反应的 结果与前者相似, 产物也是以构型保持为主(Scheme $5)^{[11]}$. 虽然从反应的立体选择性结果判断, 该类反应可 能是以 $\mathrm{S}_{\mathrm{N}} \mathrm{V}_{\pi}$ 的历程进行的, 但事实上很难判断这些反 应究竟是经历了 $\mathrm{Ad}_{\mathrm{N}}-\mathrm{E}$ 还是 $\mathrm{S}_{\mathrm{N}} \mathrm{V}_{\pi}$ 机理. 这是因为, $\beta$-卤 代苯乙烯底物中的芳香基团在一定程度上是可以稳定 反应过程中形成的碳负离子中间体的, 所以 $\mathrm{Ad}_{\mathrm{N}} \mathrm{E}$ 反应 也有可能发生, 只是所形成的碳负离子中间体的寿命太 短而导致产物主要以构型保持的形式呈现. 事实上, 以 上实验结果只发生在 $\beta$-卤代苯乙烯化合物作为反应底 物时, 而对于其他一些非芳基的烯烃, 并未发生相似的 情况.

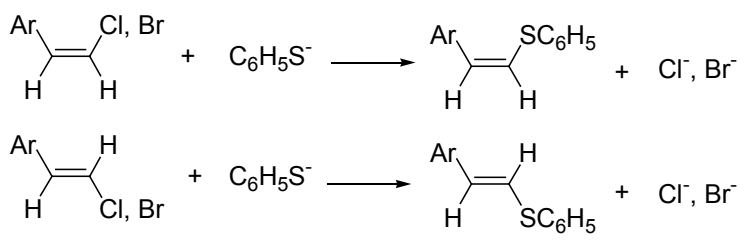

Scheme 4

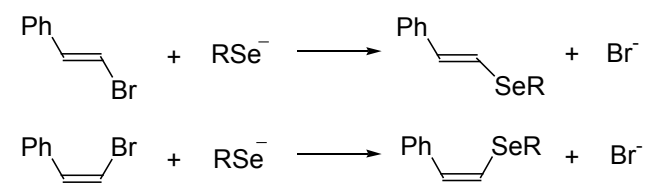

\section{Scheme 5}

此外, Apeloig 和 Rappoport ${ }^{[12]}$ 研究发现, $\beta$-取代基 $(\mathrm{Nu})$ 与碳负离子中间体上的一对电子之间的超共轭效 应在乙烯基碳的亲核取代反应的立体选择性方面(产物 的构型保持或者翻转)起到了很重要的作用(Scheme 6). 他们通过计算发现, 这种超共轭效应可以在 $\mathrm{C}-\mathrm{C}$ 键的 旋转过程中引起比较高的能垒 $\left(V_{\mathrm{x}}\right)$, 从而致使产物主要

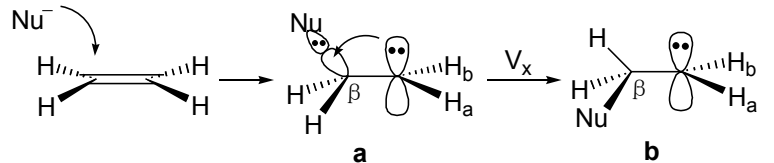

$\mathrm{Nu}=\mathrm{NH}_{2}, \mathrm{OH}, \mathrm{SH}, \mathrm{OCH}_{3}, \mathrm{~F}, \mathrm{Cl}$, etc.

$V_{\mathrm{x}}=E_{\mathrm{b}}-E_{\mathrm{a}}$ $20.2 \sim 70.1 \mathrm{~kJ} \cdot \mathrm{mol}^{-1}$

Scheme 6

以立体构型保持不变为主. 因而, 对于前面所讨论的 $\beta$ 卤代苯乙烯上的取代反应，可以认为应该是通过 $\mathrm{Ad}_{\mathrm{N}}-\mathrm{E}$ 的机理进行的: 先是硫负离子加成到双键上，卤素阴离 子紧接着迅速地消除.

\section{2 不活泼的乙烯基碳上的亲核取代反应}

真正的关于乙烯基碳上发生的亲核取代反应的报 道主要都是集中于不活泼的取代乙烯类化合物作为底 物时, 所发生的亲核取代反应才是经历 $\mathrm{S}_{\mathrm{N}} \mathrm{V}_{\sigma}$ 或者 $\mathrm{S}_{\mathrm{N}} \mathrm{V}_{\pi}$ 机理.

1991 年, Ochiai 等 ${ }^{[13]}$ 报道了第一例不活泼的乙烯基 碳上的分子间亲核取代反应. 不活泼的烯烃碘正离子盐 1 与季铵盐四丁基溴化铵中的溴离子发生取代反应生成 溴代烯烃产物, 并且产物的构型反生了翻转(Eq. 1). 这 一实验结果表明这个反应应该是通过 $\mathrm{S}_{\mathrm{N}} \mathrm{V}_{\sigma}$ 的机理进行 的, 这一推测也得到了该课题组后期的动力学研究结果 的证实 ${ }^{[14]}$.

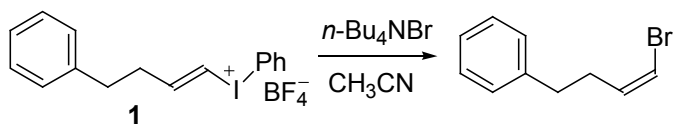

2004 年, Shipman 课题组 ${ }^{[15]}$ 报道了一例乙烯基碳上 的分子内亲核取代反应. 在一 $78{ }^{\circ} \mathrm{C}$ 条件下, $Z$ 式和 $E$ 式 的 2-溴 2-丁烯胺(2)在强碱氨基钠/液氨的作用下分别发 生环化反应生成了相应的三元环亚乙胺产物 $\mathbf{3}$, 产物的 构型发生翻转说明了氮负离子是从离去基团溴离子的 背面进攻的，也就是说该反应发生的是 $\mathrm{S}_{\mathrm{N}} \mathrm{V}_{\sigma}$ 反应 (Scheme 7).

\section{3 乙烯基碳上的分子内亲核取代反应的研究}

关于 $\mathrm{S}_{\mathrm{N}} \mathrm{V}$ 反应研究报道最多的是 Narasaka 教授课 题组 ${ }^{[16]}$. 他们研究了多种阴离子作为亲核试剂时的反 


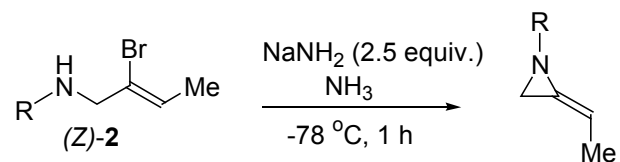<smiles></smiles>

(E)-2

a: $\mathrm{R}=\mathrm{CH}_{2} \mathrm{Ph}, \mathbf{b}: \mathrm{R}=(\mathrm{S})-\mathrm{CHMePh}$

应，包括碳负离子、氮负离子、氧负离子、硫负离子等 等. 主要讨论了乙烯基卤代物的分子内亲核取代反应, 并成功地将此类分子内 $\mathrm{S}_{\mathrm{N}} \mathrm{V}$ 反应应用到了很多杂环化 合物的合成中, 其中包括五元杂环、四元杂环以及六元 杂环等.

\section{3 .1 在五元杂环化合物中的应用}

2003 年和 2004 年, Narasaka 和 Ando 等 ${ }^{[17]}$ 通过理论 计算以及实验研究发表了一系列乙烯基卤代物的分子 内亲核取代反应. 实验结果表明: 含有分子内羟基、氨 基以及次甲基的活泼 $E$-乙烯基卤化物 $E-4 \sim E-6$ 在不同 强弱程度的碱的作用下, 都能够发生分子内亲核取代反 应, 并生成相应的五元环化合物苯并呋喃、吲哚、环戊 烯等; 但是, 其异构体 $Z$-乙烯基卤化物 Z-4 Z-6 却很难 发生反应生成相应的环化产物(Scheme 8).

根据理论计算得出, $E$-异构体反应物在经历 $\mathrm{S}_{\mathrm{N}} \mathrm{V}_{\sigma}$ 机

(E)-3 $37 \% \sim 57 \%$

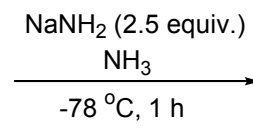

\section{Scheme 7}

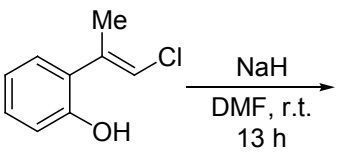

(E)-4 $\Delta G_{\mathrm{calc}}=60.5 \mathrm{~kJ} \cdot \mathrm{mol}^{-1}$<smiles>CC(=CCl)c1ccccc1N[AsH3]</smiles>

(E)-5 $\Delta G_{\text {calc }}=64.3 \mathrm{~kJ} \cdot \mathrm{mol}^{-1}$<smiles>CC(=CBr)CCC(C#N)S(=O)(=O)c1ccccc1</smiles>

(E)-6 $\Delta G_{\text {calc }}=98.3 \mathrm{~kJ} \cdot \mathrm{mol}^{-1}$<smiles>Cc1coc2ccccc12</smiles>
$95 \%$<smiles>[Y]n1cc(C)c2ccccc21</smiles>
$89 \%$

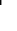<smiles>C/C(=C/Cl)c1ccccc1O</smiles>

(Z)-4

理时的过度态结构所需要的活化能较低(60.5 98.3 kJe $\mathrm{mol}^{-1}$ ); 然而理论计算发现 $Z$-异构体反应物经历 $\mathrm{S}_{\mathrm{N}} \mathrm{V}_{\pi}$ 机理比较合理, 但是仍然需要很高的活化能 $(>121.4$ $\left.\mathrm{kJ} \cdot \mathrm{mol}^{-1}\right)$, 这个计算结果合理地解释了为什么 $Z-\mathbf{4} \sim$ Z-6 很难发生相应的环化反应. 理论计算结果与前面的 实验结果是相符合的. 因而，对于含有分子内的氧、氮、 碳亲核试剂的 $E$-乙烯基卤代化合物发生的是 $\mathrm{S}_{\mathrm{N}} \mathrm{V}_{\sigma}$ 反应, 而 Z-乙烯基卤代化合物根本不发生反应.

但是，含有分子内硫醇的乙烯基溴化物 7 却表现出 了与前面的实验结果不同的现象(Scheme 9): 不仅 E-7 能够在碳酸钾的作用下以 $34 \%$ 的产率环化成为相应的 五元环化合物二氢噻吩, 其异构体 Z-7 也能够在相似的 反应条件下发生分子内亲核取代反应，并以较低的产率 生成相同的五元环产物. 通过理论计算, 对于 $E$ 式和 $Z$ 式异构体分别提出了 $S_{N} V_{\sigma}$ 和 $S_{N} V_{\pi}$ 两种机理, 因为相对 应的活化能都比较低, 分别为 52.5 和 $84.0 \mathrm{~kJ} \cdot \mathrm{mol}^{-1}$. 这 个实验结果表明，当使用硫负离子作为亲核试剂时，无 论 $E$ 式和 $Z$ 式的底物均能发生相应的分子内 $\mathrm{S}_{\mathrm{N}} \mathrm{V}$ 反应.

由于硫醇 7 很容易被氧气氧化为二硫化合物发生副 反应而影响反应的产率，为了阻止副反应的发生，提高 此类反应的产率, Narasaka 课题组 ${ }^{[18]}$ 选用了较为稳定的 硫代乙酸酯化合物 8 替代硫醇化合物 7, 在碳酸钾的作 用下，以 $\operatorname{DMI}(N, N$-二甲基咪唑)作为溶剂. 实验结果表 明: 无论是 $E-8$ 还是 Z-8 都能够顺利地发生分子内亲核 取代反应, 并以较好的产率生成二氢噻吩化合物 (Scheme 10). $\Delta G_{\mathrm{calc}}=121.4 \mathrm{~kJ} \cdot \mathrm{mol}^{-1}$

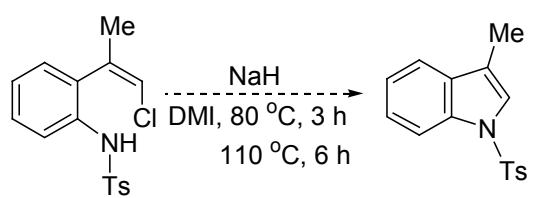

(Z)-5 Ts $\quad$ SM recovery $95 \%$ $\Delta G_{\text {calc }}=140.7 \mathrm{~kJ} \cdot \mathrm{mol}^{-1}$

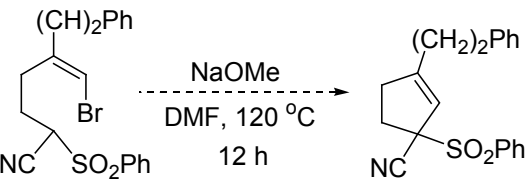

(Z)-6 $\Delta G_{\mathrm{calc}}=121.4 \mathrm{~kJ} \cdot \mathrm{mol}^{-1}$ M recovery $34 \%$ $50 \%$

[B3LYP/6-31+G(d) SCRF (dipole, DMF)]

Scheme 8 

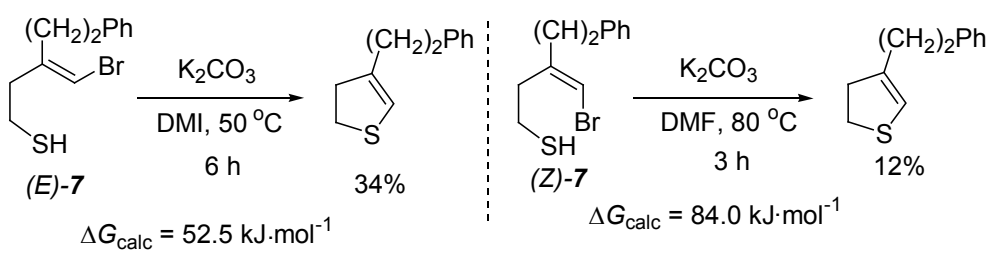

Scheme 9

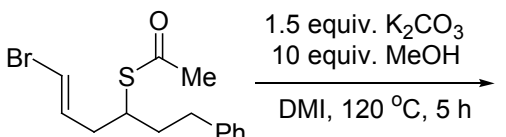

(E)-8

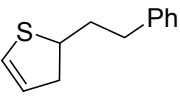

$83 \%$

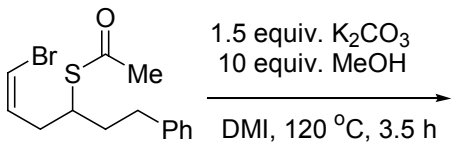

(Z)-8

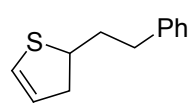

$70 \%$

\section{Scheme 10}

以上实验中所选用的反应底物都是溴原子在双键 末端的溴代化合物, 如化合物 7, 8. Narasaka 课题组 ${ }^{[18 b]}$ 也尝试合成了溴原子在双键内侧的溴代物作为底物, 如 化合物 9. 通过实验发现, 在相似的实验条件下： 1.5 equiv. 的碳酸钾，10 equiv. 的甲醇(辅助原位生成少量 $\mathrm{KOMe}$ ，增强碱性，更有利于硫负离子的产生), 在 DMI 溶剂中, 分子内的亲核取代反应也能够发生, 反应生成 五元环化合物(以 3:1 异构体的形式)(Eq. 2).<smiles>C=C(Br)CCC(CCCc1ccccc1)SC(C)=O</smiles>

$(3: 1)$

为了研究其他的类型的硫亲核试剂, 作者设计合成 了分子内含有酰胺基团的 2-溴乙烯基类化合物 $10^{[19]}$. 分子中的酰胺基团在此时可以表现为两类亲核试剂: 硫 亲核试剂和氮亲核试剂. 因而, 在此反应过程中可能发 生两种亲核进攻: 硫负离子的或者氮负离子的亲核进 攻. 但是实验结果表明, 在碳酸钾的作用下, 硫负离子 的亲核能力要强于氮负离子, 反应产物主要以硫亲核取 代产物噻唑化合物 11 为主. 因而在此基础之上, 成功地 合成出一系列含有不同取代基团的 2,5-二取代噻唑类化 合物(Eq. 3).

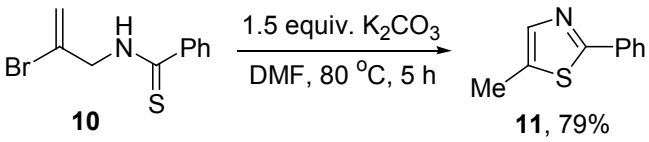

而当底物改变为分子内含有硫脲基团 12 的时候 ${ }^{[19]}$,
此时，分子中则存在另外两种亲核试剂：硫负离子亲核 试剂以及距离较远的氮负离子亲核试剂(因为距离较近 的氮原子被完全取代了). 与分子内酰胺底物 10 不同的 实验现象是：在相似的反应条件下，氮亲核试剂在此时 要强于硫亲核试剂, 即反应主要生成了氮亲核取代的产 物 3,4,5-三取代硫代咪唑类化合物 13 (Eq. 4). 我们认为 这两个反应的主要区别是由于电子效应所引起的.

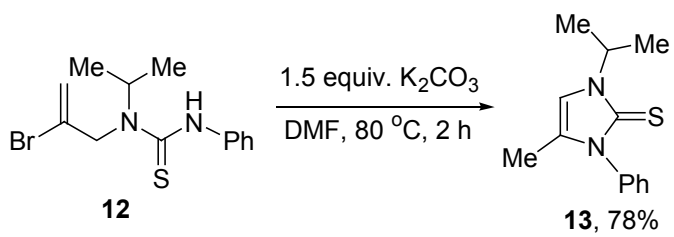

\subsection{2 在四元杂环化合物中的应用}

分子内的 $\mathrm{S}_{\mathrm{N}} \mathrm{V}$ 反应除了能成功地应用于上述五元 杂环化合物的合成，利用分子内的 $\mathrm{S}_{\mathrm{N}} \mathrm{V}$ 反应，Narasaka 课题组 ${ }^{[18 \mathrm{a}]}$ 还合成了一系列的四元环产物硫杂环丁烷 (Scheme 11). 经过理论计算发现硫负离子 15 的环化反 应如果经历 $\mathrm{S}_{\mathrm{N}} \mathrm{V}_{\sigma}$ 过渡态则需要 $93.8 \mathrm{~kJ} \cdot \mathrm{mol}^{-1}$ 的活化能, 而如果是 $S_{N} V_{\pi}$ 则要消耗 $76.2 \mathrm{~kJ} \cdot \mathrm{mol}^{-1}$. 这两个计算结 果所得到的活化能数值都不高, 因而, 很难通过理论计 算结果来判断该反应究竟经历了哪一种机理。
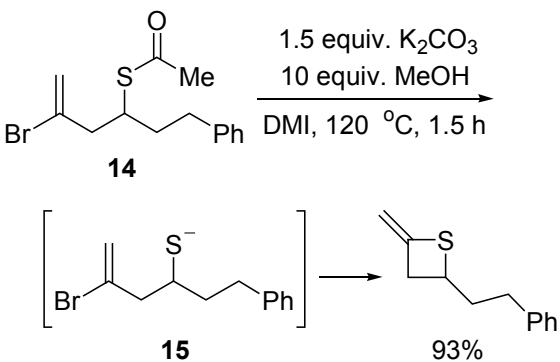

Scheme 11

为了验证以上反应究竟是经历哪种反应历程，该课 题组又进行了相关的实验研究. 利用一对异构体 Z-16 和 $E-16$, 分别在相同的实验条件下进行实验, 实验结果 表明，两种异构体均能够发生相应的环化反应，而所形 成的产物主要是构型保持为主, 即分别生成了产物 Z-17 与 $E-\mathbf{1 7}$ (Scheme 12), 因此, 通过实验证明了该类四元 环的形成应该是通过 $\mathrm{S}_{\mathrm{N}} \mathrm{V}_{\pi}$ 的机理，即产物保持构型不 变. 
<smiles>[R]C=C(Br)CC(CCc1ccccc1)SC(C)=O</smiles>

(Z)-16<smiles>[R]C(=O)SC(CCc1ccccc1)CC(=C)Br</smiles>

(E)-16

$\mathrm{R}=n-\mathrm{C}_{9} \mathrm{H}_{19}$

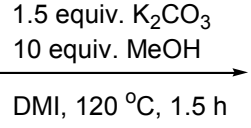

DMI, $120^{\circ} \mathrm{C}, 1.5 \mathrm{~h}$

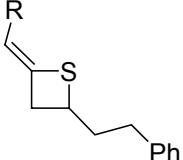

(Z)-17, 70\%

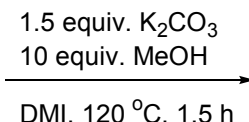

DMI, $120^{\circ} \mathrm{C}, 1.5 \mathrm{~h}$

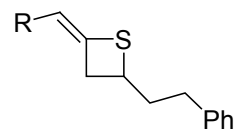

(E)-17, 62\%

\section{Scheme 12}

3.3.3 对于 $\mathrm{sp}^{2}$ 杂化碳在四元、五元及六元环关环反 应速率的比较

Casadei 等 ${ }^{[20]}$ 曾经报道过关于 $\omega$-溴代烯丙基丙二酸 酯 (18) 在碱的作用下发生环化反应的动力学研究 (Scheme 13). 一般地, 对于饱和 $\mathrm{sp}^{3}-\mathrm{C}$ 上发生的亲核取 代反应, 他们发生环化反应的速率按照碳原子数目的排 列顺序大致遵循: 五元环 $>>$ 六元环 $>$ 四元环化物.<smiles>CCOC(=O)[C@H](CCBr)[C@@H](CCBr)[C@H](CCBr)C(OCC)OCC</smiles>

18

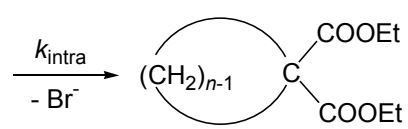

$n=5>6>4$

$k_{\text {intra }}=6 \times 10^{2} \mathrm{~s}^{-1}>0.72 \mathrm{~s}^{-1}>0.42 \mathrm{~s}^{-1}$

\section{Scheme 13}

然而, 对于不饱和 $\mathrm{sp}^{2}-\mathrm{C}$ 的分子内环化反应的速率 却无报道. 因此, 选用比化合物 9 多一个碳原子的底物 19, 在相似的反应条件之下, 六元环的环化反应也能够 发生, 但是反应的产物不仅仅是六元环产物(不可分离 的异构体混合物), 还发现了炔烃化合物的生成(原料经 脱溴化氢得到)(Eq. 5 ${ }^{[18 \mathrm{~b}]}$. 这一实验结果与前面报道的 五元环化相比, 对于 $\mathrm{sp}^{2}-\mathrm{C}$ 发生的亲核反应来说, 五元 环化物的形成更显容易.

另外, 为了比较四元环与五元环的环化反应的优先 次序，他们还设计合成了一个含有两个 2-溴乙烯基的化 合物 20, 使得亲核试剂硫负离子与离去基团溴之间分 别相间了三个和四个碳原子数目, 使反应既有环化成为 四元环、也可以成为五元环的可能. 事实上, 反应结束 后, 只分离得到了四元的环化产物硫杂环丁烷 21, 却没 有相应的五元环产物生成(Scheme 14) ${ }^{[18 b]}$. 这一实验结 果说明了对于 $\mathrm{sp}^{2}-\mathrm{C}$ 的发生的分子内硫负离子亲核取代 反应，四元环的形成要优先于五元环.

那么, 与前一实验结果相结合, 对于 $\mathrm{sp}^{2}-\mathrm{C}$ 的环化

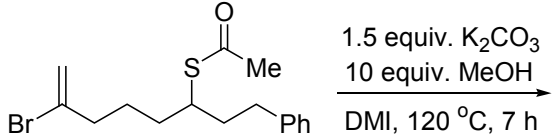

19<smiles>C=C1CCCC(CC[PH2+]c2ccccc2)S1</smiles>

$31 \%(2.5: 1)$<smiles>C#CCCCC(CCc1ccccc1)SC(C)=O</smiles>

$19 \%$<smiles>C=C(Br)CCC(CC(=C)Br)SC(=O)O</smiles>

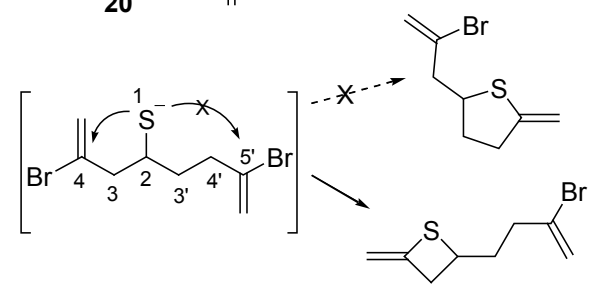

$21,87 \%$

Scheme 14

反应的速率比较大致可以得出这么一个粗略的结论：四 元环 $>$ 五元环 $>$ 六元环化物(Eq. 6).

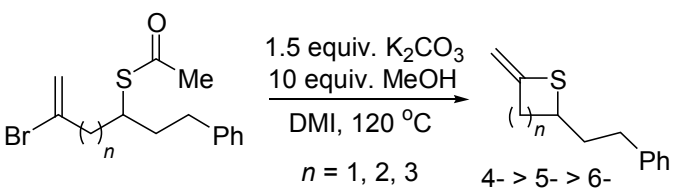

从以上如此多实验事实和理论计算结论来看，分子 内乙烯基碳上的亲核取代反应 $\left(\mathrm{S}_{\mathrm{N}} \mathrm{V}\right)$ 是可以实现的，并 且能够为合成不同的杂环化合物提供一个独特的渠道.

\section{$4 \mathrm{sp}^{2}$ 杂化氮上的亲核取代反应}

亲核取代反应不仅能够发生在 $\mathrm{sp}^{2}-\mathrm{C}$ 上, 而且还能 够发生在 $\mathrm{sp}^{2}-\mathrm{N}$ 上. 事实上, Narasaka 课题组 ${ }^{[21]}$ 关于乙烯 基碳上的分子内亲核取代反应的研究就是在 $\mathrm{sp}^{2}-\mathrm{N}$ 上的 亲核取代反应的基础之上展开的.

1995 年, 在研究肜化合物 22 的贝克曼催化重排反 应的时候，不仅发现了贝克曼重排反应的产物酰胺 23 , 而且还分离出一个意外的产物喹啉 20 (Eq. 7).

更进一步的实验与理论计算证实了这个意外产物 喹啉 27 是通过 $\mathrm{sp}^{2}-\mathrm{N}$ 上的亲核取代反应 $\left(\mathrm{S}_{\mathrm{N}} \mathrm{V}_{\sigma}\right)$ 生成的. 通过机理研究发现 ${ }^{[22]}$ : 肟 25 在被四丁基高铼酸铵催化 下，首先肟羟基被转变成为酯，进一步发生了苯环的平 

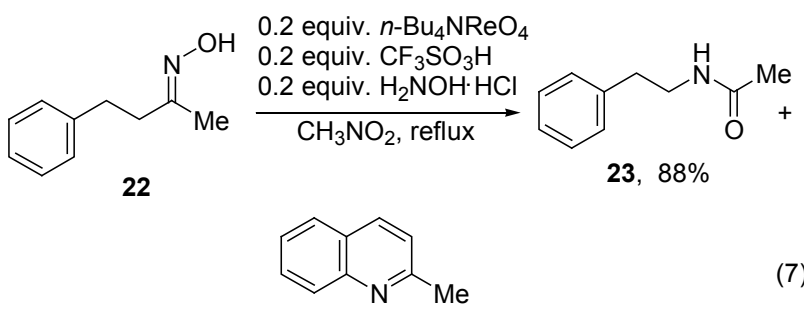

24, $7 \%$

面内进攻 $\mathrm{sp}^{2}-\mathrm{N}$ 从而形成了一个螺环中间体 26. 紧接着, 中间体 26 经过重排反应生成喹啉化合物 27 (Scheme $15)$. 并且, 理论计算进一步得出, $\mathrm{sp}^{2}-\mathrm{N}$ 上发生亲核取代 反应所需要的活化能 $\left(37.0 \mathrm{~kJ} \cdot \mathrm{mol}^{-1}\right)$ 与发生贝克曼重排 反应所需的能量 $\left(33.6 \mathrm{~kJ} \cdot \mathrm{mol}^{-1}\right)$ 是几乎相等的 ${ }^{[23]}$.

利用 $\mathrm{sp}^{2}-\mathrm{N}$ 上的分子内或者分子间的亲核取代反应，
可以合成出很多含氮杂环化合物，包括吡咯化合物、四 氢吡啶类以及胺类化合物等等 $(\text { Scheme 16 })^{[24,25]}$.

\section{5 结论与展望}

尽管 $\mathrm{sp}^{2}$ 杂化碳上发生的亲核取代反应在过去很长 一段时间内被有机化学研究者们认为是很难发生的, 但 是近 20 年来的理论研究以及实验结果均显示, 此类反 应是可以发生的. 利用乙烯基碳上的亲核取代反应，特 别是乙烯基碳上的协同分子内亲核取代反应(分子内 $\left.\mathrm{S}_{\mathrm{N}} \mathrm{V}\right)$, 能够合成出一系列地含有不同取代基团的杂环 类化合物，包括四元、五元以及六元环化合物等. 作者 相信, 此类反应将在以后的有机化学发展过程中得到越 来越多的重视和应用.

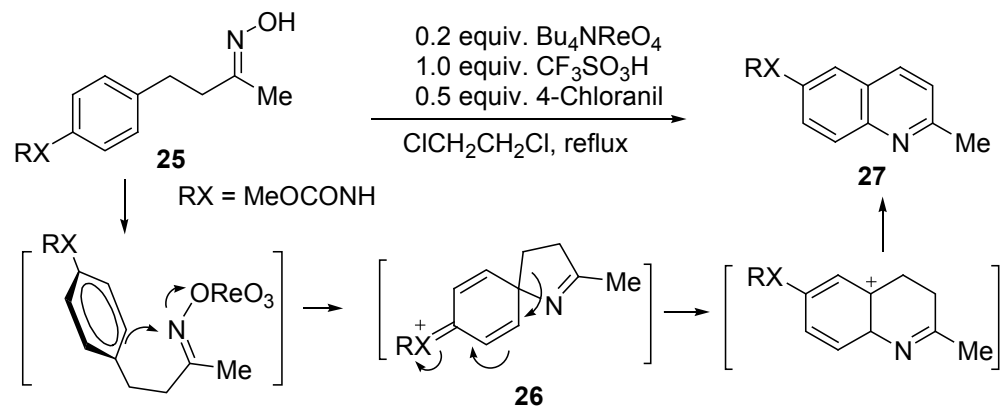

\section{Scheme 15}<smiles>CO[R6]O/N=C(\C)CC(C(C)=O)c1ccccc1</smiles>
$\underset{\mathrm{CH}_{2} \mathrm{Cl}_{2}, 0^{\circ} \mathrm{C}}{\mathrm{DBU}}$ $\mathrm{OSO}_{2} \mathrm{Me}$

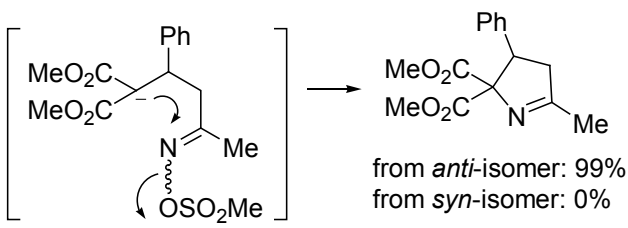

\section{Scheme 16}

\section{References}

[1] (a) Smith, M.; March, J. March's Advanced Organic Chemistry, 6th ed., Wiley-Interscience, New York, 2007, p. 425.

(b) Hartshorn, S. R. Aliphatic Nucleophilic Substitution, Cambridge University Press, London, 1973.

(c) Katritzky, A. R.; Brycki, B. E. Chem. Soc. Rev. 1990, 19, 83.

[2] Mayr, H.; Minegishi, S. Angew. Chem., Int. Ed. 2002, 41, 4493.

[3] Cowdrey, W. A.; Hughes, E. D.; Ingold, C. K.; Masterman, S.; Scott, A. D. J. Chem. Soc. 1937, 1252.

[4] Terrier, F. Modern Nucleophilic Aromatic Substitution, Wiley-VCH, Weinheim, 2013.

[5] (a) Smith, M.; March, J. March's Advanced Organic Chemistry, 6th ed., Wiley-Interscience, New York, 2007, p. 481.

(b) Wells, P. R. Prog. Phys. Org. Chem. 1968, 6, 111.

[6] (a) Rappoport, Z. Acc. Chem. Res. 1981, 14, 7.

(b) Rapport, Z. Acc. Chem. Res. 1992, 25, 474.

(c) Miller, S. I.; Yonan, P. K. J. Am. Chem. Soc. 1957, 79, 5931.

(d) Modena, G. Acc. Chem. Res. 1971, 4, 73.

(e) Miller, S. I. In Advances in Physical Organic Chemistry, Vol. 6, Ed.: Gold, V., Academic, New York, 1968, 185.
[7] (a) Bach, R. D.; Baboul, A. G.; Schlegel, H. B. J. Am. Chem. Soc. 2001, 123, 5787.

(b) Lucchini, V.; Modena, G.; Pasquato, L. J. Am. Chem. Soc. 1995, 117, 2297.

[8] Glukhovtsev, M. N.; Pross, A.; Radom, L. J. Am. Chem. Soc. 1994, 116, 5961.

[9] Kim, C. K.; Hyun, K. H.; Kim, C. K.; Lee, I. J. Am. Chem. Soc. 2000, 122, 2294.

[10] (a) Marchese, G.; Naso, F.; Modena, G. J. Chem. Soc. B 1968, 958. (b) Marchese, G.; Naso, D.; Modena, G. J. Chem. Soc. B 1969, 290.

[11] Tiecco, M.; Testaferri, L.; Tingoli, M.; Chianelli, D.; Montanucci, M. Tetrahedron Lett. 1984, 25, 4975.

[12] (a) Hoffmann, R.; Radom, L.; Pople, J. A.; Schleyer, P. V. R.; Hehre, W. J.; Salem, L. J. Am. Chem. Soc. 1972, 94, 6221.

(b) Apeloig Y.; Rappoport, Z. J. Am. Chem. Soc. 1979, 101, 5095. (c) Miller, S. I. Tetrahedron 1977, 33, 1211.

[13] (a) Ochiai, M.; Oshima, K.; Masaki, Y. J. Am. Chem. Soc. 1991, 113, 7059 .

(b) Ochiai, M.; Nishi, Y.; Hirobe, M. Tetrahedron Lett. 2005, 46, 1863.

[14] Okuyama, T.; Takino, T.; Sato, K.; Ochiai, M. J. Am. Chem. Soc. 
1998, 120, 2275.

[15] Shiers, J. J.; Shipman, M.; Hayes, J. F.; Slawin, A. M. Z. J. Am. Chem. Soc. 2004, 126, 6868.

[16] Chiba, S.; Ando, K.; Narasaka, K. Synlett 2009, 2549.

[17] (a) Ando, K.; Kitamura, M.; Miura, K.; Narasaka, K. Org. Lett. 2004, 6, 2461.

(b) Miyauchi, H.; Chiba, S.; Fukamizu, K.; Ando, K.; Narasaka, K. Tetrahedron 2007, 63, 5940.

[18] (a) Lei, M.-Y.; Fukamizu, K.; Xiao, Y.-J.; Liu, W.-M.; Twiddy, S.; Chiba, S.; Ando, K.; Narasaka, K. Tetrahedron Lett. 2008, 49, 4125 .

(b) Lei, M.-Y.; Xiao, Y.-J.; Liu, W.-M.; Fukamizu, K.; Chiba, S.; Ando, K.; Narasaka, K. Tetrahedron 2009, 65, 6888.

[19] Shen, S.-S.; Lei, M.-Y.; Wong, Y.-X.; Tong, M.-L.; Teo, P.; Chiba, S.; Narasaka, K. Tetrahedron Lett. 2009, 50, 3161.

[20] Casadei, M. A.; Galli, C.; Mandolini, L. J. Am. Chem. Soc. 1984, $106,1051$.
[21] Narasaka, K.; Kitamura, M. Eur. J. Org. Chem. 2005, 4505.

[22] Kusama, H.; Yamashuta, Y.; Narasaka, K. Chem. Lett. 1995, 5.

[23] Mori, S.; Uchiyama, K.; Hayashi, Y.; Narasaka, K.; Nakamura, E. Chem. Lett. 1998, 111.

[24] (a) Kusama, H.; Uchiyama, K.; Yamashita, Y.; Narasaka, K. Chem. Lett. 1995, 715.

(b) Kusama, H.; Yamashita, Y.; Uchiyama, K.; Narasaka, K. Bull. Chem. Soc. Jpn. 1997, 70, 965.

(c) Uchiyama, K.; Yoshida, M.; Hayashi, Y.; Narasaka, K. Chem. Lett. 1998, 607.

[25] (a) Yoshida, M.; Uchiyama, K.; Narasaka, K. Heterocycles 2000, 52,681 .

(b) Kitamura, M.; Yoshida, M.; Kikuchi, T.; Narasaka, K. Synthesis 2003, 2415.

(c) Tanaka, K.; Mori, Y.; Narasaka, K. Chem. Lett. 2004, 33, 26.

(d) Kitamura, M.; Suga, T.; Chiba, S.; Narasaka, K. Org. Lett. 2004, 6, 4619 . 